This is the pre-publication manuscript of:

Shelton C. (2017) How Can We Make Computing Lessons More Inclusive? In: Tatnall A., Webb M. (eds) Tomorrow's Learning: Involving Everyone. Learning with and about Technologies and Computing. WCCE 2017. IFIP Advances in Information and Communication Technology, vol 515. Springer, Cham. pp. 506-514

https://doi.org/10.1007/978-3-319-74310-3

\title{
How Can We Make Computing Lessons More Inclusive?
}

\author{
Chris Shelton \\ Institute of Education, University of Chichester, UK \\ c.shelton@chi.ac.uk
}

\begin{abstract}
Whilst there is a substantial body of research that shows how Information and Communications Technologies (ICTs) can support schools and teachers to make their classrooms more inclusive, there is a need for more evidence describing how best to ensure that the teaching of computing itself is inclusive. This paper reports on a literature review of inclusive education in school computing lessons. It identifies a number of inclusive practices, including ensuring a relevant and authentic curriculum that focuses on depth of understanding, promoting culturally relevant tasks, and ensuring an inclusive environment that challenges bias. The review also identifies a need for much more research into what constitutes an inclusive computing classroom.
\end{abstract}

Keywords. Schools, Computing, Computer Science Education, Inclusion, Pedagogy

\section{Introduction}

According to UNESCO [1], inclusion is "a process of addressing and responding to the diversity of needs of all children, youth and adults through increasing participation in learning, cultures and communities, and reducing and eliminating exclusion within and from education" (p8). An inclusive school is therefore one which modifies its "content, approaches, structures and strategies" (p9) to better educate all children.

While early debates about inclusion focused on whether pupils should be educated in mainstream schools or alternative 'special' provision [2], over time, understandings of inclusion have broadened away from the location of schooling to address a wide range of pupil characteristics, including, but not limited to: race, class, gender, language, cultural and social differences. Ainscow, Booth and Dyson [3] suggest that inclusion is an approach to education based on inclusive values (including equity, participation, compassion, respect of diversity, sustainability and entitlement).

School pupils are not a homogenous group [4] and there is now a substantial body of research that shows how the most effective uses of Information and Communications Technologies (ICTs) can support schools and teachers to make their classrooms more inclusive (see, for example, Seale [5]). However, while much research on use of ICT for inclusion is relevant to all teachers, there are still gaps in the evidence of how best to ensure that computing lessons are inclusive. Given the 
concern over low participation rates in Higher Education and the later years of secondary computing, there is a need for specific research that investigates how inclusive school teaching of computing is and how this might be improved. This is vital as there is evidence that digital literacy is highly influenced by inequalities outside of school [4] and that a lack of access and skills forces individuals and groups to becoming uncritical consumers of digital texts [6].

Therefore, this literature review was intended to survey the current literature regarding inclusive computing education in schools to identify both aspects of effective practice and areas for further research.

\section{Method}

The aim of this project was to conduct a literature review of research focusing on inclusive computing teaching in schools. There were three criteria for inclusion in the review:

1) The text must be concerned with school level education

2) The text must focus on the teaching of computing or computer science (rather than the use of technology across the curriculum)

3) The text must focus on one or more issues of inclusion

In order to find sources for the review, three databases were searched: ERIC (eric.ed.gov), Web of Science (including the Social Sciences Citation Index (SSCI) and Conference Proceedings Citation Index - Social Science and Humanities (CPCI$\mathrm{SSH})$ ) and the ACM Digital Library Guide to Computing Literature. The ACM database contains both full-text and citation data regarding computer science research as well as social science research and therefore an adapted search strategy was required for this database. The searches were conducted in January 2017.

Three categories of search terms were required to ensure that the three criteria were met:

1) To ensure that all texts were focussed on school level education, the terms searched for were: "primary", "secondary", "elementary", "school" or "K12 ". For the ACM database, the terms "school" and "primary" were excluded as they returned texts relating to Higher Education and/or Computer Science.

2) To ensure that all texts were focussed on the teaching of computing, the following terms were used: "computing", "teaching IT", "teaching ICT", "teaching information technology", "teaching information communication technology", "computer science", "computer programming", "digital literacy" and "informatics".

Other terms considered but not used included "coding" (this gave irrelevant results due to the use of coding as a term to describe analyse of research data), "programming" (frequently used to refer to the design of special education intervention programmes) and variations of "IT" (these led to several thousand papers many of which were about the use of technology to 
teach across the curriculum).

This search term was not required for the ACM database as all texts were related to computing in some form.

3) To ensure the texts focus on issues of inclusion, the terms "inclusive" or "inclusion" were used. (Where allowable, the wildcard inclus* was used.)

The initial searches resulted in 329 possible sources. The abstracts of all texts identified by these searches were read and assessed against the three criteria. This resulted in 29 relevant publications. The relatively high rate of ineligible publications reflects the multiple meanings of the search terms.

Table 1. Summary of search results

\begin{tabular}{lll}
\hline Database & Potential sources & Sources meeting criteria \\
\hline ERIC & 33 & 10 \\
SSCI / CPCI-SSH & 127 & 7 \\
ACM & 169 & 12 \\
\hline
\end{tabular}

\section{Results and Discussion}

The papers identified through this review identify several specific inclusion issues: gender, diversity, special educational needs, attainment, and economic disadvantage. While these issues are reported separately below and the majority of papers had a clear focus on just one of these, some papers acknowledged that these issues intersect. However, none of the papers attempted to provide a comprehensive description or analysis of inclusive computing teaching or pedagogy.

\section{Gender}

The most commonly discussed inclusion topic was gender. There is clear evidence that females are significantly underrepresented in computing (both in employment and in the proportion taking higher qualifications) [7]. Ashcraft et al. provides a comprehensive literature review of the research into underrepresentation of females in computing [7]. This review shows that girls' perceptions, interests, confidence and career decisions are influenced by many factors and categorises these factors as formal/informal education, peer influences, families and community influences and media influences. Of particular relevance for this review are the sections pertaining to formal education where Ashcraft et al. identify issues of curriculum and pedagogy.

In terms of curriculum, Ashcraft et al. suggest that girls may often hold perceptions that the computing curriculum is irrelevant to them. They suggest that computing has often been taught in the abstract rather than addressing how computing can help to address real-life problems. This abstract curriculum also reinforces views of computing as a "lonely, isolated, machine-focused set of tasks" (p21). 
The review highlights the work of Lasen [8] that compares the views of school girls who chose or did not choose to take advanced ICT options. Both those who choose these courses and those who did not both expressed "an aversion to programming" with those who took the courses attracted to the creative aspects of the subjects (such as multimedia and web design) and enjoying authentic, problem based tasks. The view that girls do not opt for computing subjects because they do not value them is also expressed by Downes and Looker [9].

This suggests that a computing curriculum needs to be designed to incorporate and connect to real-life problems. It should also address some of the other areas that influence pupil perceptions, for example, by incorporating lessons on or discussions of media representations of computing.

Another curriculum issue identified by Ashcraft et al. is a focus on breadth of coverage and speed rather than depth and fluency. A computing curriculum that is focussed on speed and competence at isolated tasks can negatively affect pupils who prefer to study more deeply [7]. They suggest that computing curricula should focus on fluency - activities that transfer or can be applied in other contexts.

One suggestion proposed as a way of engaging girls has been a curriculum based around computer games. However, Osunde et al. [10] have identified that teenage boys and girls prefer games with different features (e.g. graphical style, storyline, number of players). While Robertson [11] provides an example of a game making project that was enjoyed by students but did not address the gender gap and even indicated that pupils were "less likely to like computing, or want to find out more about the subject after taking part in the project" (p37).

The second set of issues relate to pedagogy and Ashcraft et al. suggest that handson activities, collaborative work, and project-based learning are important to motivate and include girls. They suggest that active, practical work retains pupils' interest and help to make computing relevant while improving fluency. Similarly, Corneliussen, and Proitz [12] suggest that school computing curricula need to adopt the playfulness with technology found in after school code clubs in order to include girls.

Ashcraft et al. also suggest that there is evidence that the use of collaborative learning activities have positive effects for girls, including increased persistence in the face of problems. However, they claim that teachers tend to privilege independent work and sometimes even discourage group-work.

It is also important to ensure that the classroom environment promotes suitable role models and avoids stereotypical displays (Ashcraft et al. mention environments referencing science fiction and displaying computer parts as being less appealing for girls [7]). Coupal [13] highlights the importance of paying attention to power relationships in school and Ashcraft et al. suggest that teachers need to pay attention to classroom interactions to ensure pupils receive reliable feedback and avoid pupils judging themselves based on their speed of work rather than depth of understanding.

Ashcraft et al. also suggest that teachers need to be aware of the potential for unconscious bias regarding who will be most likely to succeed in computing and warns against mistaking prior experience for innate ability. As Downes and Looker [9] note, ability and value beliefs are gendered. Fisher and Cox [14] note that programming contests use assessment methods that are biased towards males, (e.g. 
through time constraints, question topics, etc.) and this may have implications for multiple choice assessments in schools.

\section{Diversity}

Ladner and Israel [15] point out that in the USA, pupils of school age are ethnically diverse with less than $50 \%$ of the population white and $15 \%$ of students having a disability. This highlights the need for teachers to "be able to implement pedagogies that promote learning and engagement for diverse learners" (p27). Iqbal et al [16] note that there has been limited research into minority ethnic pupils' experiences of computing in the UK. In their study, "pupils suggested they were more engaged when teachers tried to overcome the disconnection with pupils' social context outside of school" (p1297).

Ladner and Israel suggest that one potential approach to including diverse learners is "culturally responsive computing education". This is described by Eglash, Gilbert and Foster [17] as approaches which merge computational thinking with cultural practices. This might include demonstrating the sophisticated mathematical or computational practices found in pupils' cultural heritage; using culturally authentic characters as sprites when teaching Scratch; or engaging in projects related to culturally relevant problems or issues.

Promoting culturally relevant activities is suitable for all ages of school pupils. Lotherington and Chow [18] describe how very young pupils created culturally relevant multimedia versions of the traditional tale, Goldilocks, thus developing digital literacy skills in a culturally diverse way. While Pirbhai-Illich, Turner and Austin [6] show that high school students in alternative education provision can create multimedia texts reflecting their culture.

\section{Special Educational Needs}

Pupils with Special Educational Needs are also at risk of exclusion from computing lessons. Ladner and Israel [15] identify three aspects of exclusion: teachers' attitudes/expectations; pedagogical approaches; and accessibility.

Teachers' attitudes can have a negative impact on pupils' achievement in computing if they hold lower expectations of a pupil because of their particular needs. Ladner and Israel suggest that this may "create an unconscious bias that the student cannot learn computer science" (p27). In contrast, Echeveste [19] shows that this is not necessarily the case and observed that "students labeled as special ed worked steadily on programming tasks performing sometimes better than their peers" (p359). However, Echeveste does not explain why this might be or if particular features of the tasks or pedagogy supported this.

For pupils with certain Special Educational Needs, specific resources or approaches are required to enable them to access the curriculum. For example, pupils with visual impairment remain underrepresented in higher education computing [20]. In recent years, there has been a huge rise in the use of block-based programming languages, e.g. Scratch, particularly to introduce computing to pupils. While there are numerous benefits to such languages, they are not accessible for pupils with visual impairments [21]. In an outreach robotics program for secondary pupils with visual 
impairments, inclusive practices that would be appropriate for all subjects (e.g. use of braille, allowing pupils time to orient themselves to the space, etc.) were supplemented by practices that were specific to computing: the use of a text based language, fully commented source code and a screen reader set to read all punctuation [20]. Together these supported pupils to solve complex challenges. Another approach is that of Papazafiropulos et al. [22] who used 3D printing to produce accessible resources to teach visually impaired pupils computer science concepts (e.g. data structures). They found that the resources they created were also successful with sighted pupils and had potential to be used in inclusive classrooms

\section{Attainment}

In any computing class, there will also be differences in pupils' level of attainment in computing. These differences may relate to other aspects of inclusion. For example, Hatlevik and Christophersen [23] suggest that some of the differences between pupils' digital competence relate to their cultural capital and home language. Others note that perceptions and beliefs about ability are often biased and gendered [9].

Even when differences of attainment are identified, teachers may not be equipped to ensure that the work they set is at the correct level of challenge for pupils. As Dagiene and Stupuriene [24] note, the experience of question authors for the Bebras computing competition has shown that experts do not evaluate accurately the difficulty and complexity of the tasks they set for different age groups.

It is noticeable that none of the reviewed papers fully addressed how teachers can support the wide range of attainment that might be expected in a school computing class. There is clearly scope for adapting the inclusive pedagogical approaches used successfully in many other subjects [25] and for researchers to engage with work on pupil mindsets and alternatives to ability grouping and setting. For example, through setting a range of different learning challenges that demonstrate progression in the aspect of computing being taught, children can select for themselves the task that is at the most appropriate level for them [26].

\section{Economic disadvantage}

Few of the reviewed papers specifically addressed ways of including pupils who have less access to technology because of economic disadvantage. As Ashcraft et al. noted above, attainment in computing may have more to do with prior experience than any 'natural' ability and Iqbal et al. [16] show that insufficient access to technology can be related to the socio-cultural learning contexts of minority groups. Thus, inequalities are compounded with this limited access having a more acute impact for pupils who are already disadvantaged in other ways.

There are examples of specific actions taken to address digital exclusion. Thomaz et al. [27] demonstrate how practical activities with robotics motivated and enriched the learning of digitally excluded pupils in Brazil. These activities demonstrated several features of pedagogy identified by researchers looking at other areas of inclusion, for example, using practical activities and valuing collaboration 


\section{Conclusions}

This review found research relating to a number of inclusion issues, in particular, gender, diversity, special educational needs, attainment and economic disadvantage. However, the number of papers found was relatively small due to the use of the search terms "inclusion" and "inclusive". It is clear that there are other studies relating to each of the five areas above that do not use these terms and so do not appear in this review, for example, there was little reference to research that considers the experiences of pupils with autism learning computing, or parental influences on young peoples' participation in computing. The gaps in the review are indicative of the way that research in each of these areas has remained separate from the wider educational literature on inclusion and inclusive pedagogies or even to the literature on the use of ICT for inclusion. The review has shown that there is insufficient research that synthesises these different strands and provides the guidance that teachers need to ensure that their computing lessons are truly inclusive for all pupils.

That is not to say that researchers do not recognise the need for work that addresses the full range of inclusion issues and particularly focuses on those who are disadvantaged in multiple ways. In fact, Ashcraft et al. [7] suggests that a crucial next step in efforts to diversify computing is "future research that takes a more in-depth look at intersections of race, gender, class, ability, and sexuality" (p5).

Notwithstanding these limitations, the review has identified some common practices that have potential to further the inclusion of underrepresented and disadvantaged groups by improving the school computing curriculum, teachers' pedagogy or individual attitudes. Firstly, there is a need to ensure that the school computing curriculum is relevant and authentic. Rudd [28] demonstrates how recent changes to the ICT curriculum in England reflect ideological and neo-liberal political positions which could lead to computer science being constructed as a selective and elitist subject. In contrast, he argues the need for a curriculum and practices that promote greater social justice and equity. Such a curriculum must be designed so that it is valued by pupils and addresses issues that are important to them. One such approach is to focus computing lessons on developing 'computational participation' which Kafai and Burke propose as an extension of the concept of computational thinking to include personal expression and social participation [29]. Approaches to computing should emphasise deep fluency rather than superficial understanding and speed and highlight the social aspects of computing rather than reinforce stereotypes of the lone, isolated programmer. For example, Holbert demonstrates how creating artefacts for others may help to align computing with values of connectedness [30]. This can be complemented by "culturally responsive computing" approaches that purposefully value and engage with aspects of pupils' culture or go further and actively promote critical reflection and engagement with issues of exclusion, disadvantage and power relationships (see, for example, the work of Lee and Soep [31]).

Such a curriculum should be taught through inclusive pedagogical approaches that allow children to engage in meaningful practical tasks in a welcoming environment. Teachers should encourage collaboration and pay attention to potential unconscious 
bias and how interactions within the classroom may promote or reinforce stereotypical behaviour.

In terms of future research, there is a need for a more comprehensive review of each of the five themes identified here that draws on the wider literature of inclusive practice and applies this to computing. It is also clear that many studies of inclusion in computing have been situated in informal or outreach activities rather than inclusive mainstream classrooms. As Kafai and Burke point out, extra-curricular activities compete with pupils' other interests and will never reach all of the pupils who may benefit from them [29]. Therefore, schools have a vital role to play in ensuring all pupils have an inclusive experience of computing and researchers have an important role in supporting this by researching computing practices in more formal settings.

\section{References}

1. UNESCO, Policy Guidelines on Inclusion in Education. 2009, UNESCO: Paris.

2. Frederickson, N. and T. Cline, Special Educational Needs, Inclusion and Diversity. 2nd Edition. 2009, Maidenhead: Open University Press.

3. Ainscow, M., T. Booth, and A. Dyson, Improving schools, developing inclusion. 2006, London: Routledge.

4. Meneses, J. and J.M. Momino, Putting Digital Literacy in Practice: How Schools Contribute to Digital Inclusion in the Network Society. Information Society, 2010. 26(3): p. 197-208.

5. Seale, J., Digital Inclusion: A Research Briefing by the TechnologyEnhanced Learning Phase of the Teaching and Learning Research Programme, in London Knowledge Lab, London,[online] Available at: http://www.tlrp.org/docs/DigitalInclusion.pdf. 2010.

6. Pirbhai- Illich, F., K.C. Nat Turner, and T.Y. Austin, Using digital technologies to address Aboriginal adolescents' education. Multicultural Education \& Technology Journal, 2009. 3(2): p. 144-162.

7. Ashcraft, C.S., E. Eger, and M. Michelle Friend, Girls in IT. 2013, NCWIT.

8. Lasen, M., Education and career pathways in Information Communication Technology: What are schoolgirls saying? Computers \& Education, 2010. 54(4): p. 1117-1126.

9. Downes, T. and D. Looker, Factors that influence students' plans to take computing and information technology subjects in senior secondary school. Computer Science Education, 2011. 21(2): p. 175-199.

10. Osunde, J., et al. An Investigation of Digital Games Features That Appeal to Young Females and Males. in Proceedings of the 9th European Conference on Games Based Learning. Ed. R. Munkvold and L. Kolas. 2015.

11. Robertson, J., The Influence of a Game-Making Project on Male and Female Learners' Attitudes to Computing. 2013. 23(1): p. 58-83.

12. Corneliussen, H.G. and L. Prøitz, Kids Codein a rural village in Norway: could code clubs be a new arena for increasing girls' digital interest and 
competence? Information, Communication \& Society, 2015. 19(1): p. 95110 .

13. Coupal, L.V., Computer-based Technologies and the Social Construction of Gendered Identities: An Ethnography of the Power/Knowledge Relations of Schooling. PhD Thesis. 2003.

14. Fisher, M. and A. Cox, Gender and Programming Contests: Mitigating Exclusionary Practices. Informatics in Education, 2006. 5(1): p. 1-16.

15. Ladner, R.E. and M. Israel, "For all" in "computer science for all". Communications of the ACM, 2016. 59(9): p. 26-28.

16. Iqbal, J., et al., The face of digital literacy for Muslim teenage girls: a comparative study of Bradford Muslim girl schools. International Journal of Inclusive Education, 2014. 18(12): p. 1283-1303.

17. Eglash, R., J.E. Gilbert, and E. Foster, Toward culturally responsive computing education. Communications of the ACM, 2013. 56(7): p. 33.

18. Lotherington, H. and S. Chow, Rewriting "Goldilocks" in the Urban, Multicultural Elementary School. The Reading Teacher, 2006. 60(3): p. 244252.

19. Echeveste, M.E., Challenges of Introducing Computer Science into the Traditional Grammar of K-12 Schooling. 2016: p. 359-359.

20. Ludi, S. and T. Reichlmayr, Developing Inclusive Outreach Activities for Students with Visual Impairments. Sigcse'08: Proceedings of the 39th ACM Technical Symposium on Computer Science Education, 2008: p. 439-443.

21. Ludi, S. and T. Reichlmayr, The Use of Robotics to Promote Computing to Pre-College Students with Visual Impairments. ACM Transactions on Computing Education, 2011. 11(3): p. 1-20.

22. Papazafiropulos, N., et al., Haptic Models of Arrays Through 3D Printing for Computer Science Education. 2016. 9758: p. 491-498.

23. Hatlevik, O.E. and K.-A. Christophersen, Digital competence at the beginning of upper secondary school: Identifying factors explaining digital inclusion. Computers \& Education, 2013. 63: p. 240-247.

24. Dagiene, V. and G. Stupuriene, Bebras - a Sustainable Community Building Model for the Concept Based Learning of Informatics and Computational Thinking. Informatics in Education, 2016. 15(1): p. 25-44.

25. Florian, L., Conceptualising Inclusive Pedagogy: The Inclusive Pedagogical Approach in Action. in Joanne M. Deppeler , Tim Loreman , Ron Smith , Lani Florian (ed.) Inclusive Pedagogy Across the Curriculum (International Perspectives on Inclusive Education, Volume 7) 2015 Emerald Group Publishing Limited, pp.11 - 24

26. Shelton, C. Beyond lesson recipes: first steps towards a repertoire for teaching primary computing. in Proceedings of Constructionism 2016. Bangkok.

27. Thomaz, S., et al. RoboEduc: A Pedagogical Tool to Support Educational Robotics. in 39th ASEE/IEEE Frontiers in Education Conference. 2009.

28. Rudd, T., The ideological construction of a new form of digital exclusion: computer science as latin or total deus ex machina? The Journal for Critical Education Policy Studies, 2013. 11(3): p. 287-305. 
29. Kafai, Y.B. and Q. Burke, Connected code: Why children need to learn programming. 2014, Boston: MIT Press.

30. Holbert, N., Leveraging cultural values and "ways of knowing" to increase diversity in maker activities. International Journal of Child-Computer Interaction, 2016. 9-10: p. 33-39.

31. Lee, C.H. and E. Soep, None But Ourselves Can Free Our Minds: Critical Computational Literacy as a Pedagogy of Resistance. Equity \& Excellence in Education, 2016. 49(4): p. 480-492. 